POS $\quad \begin{aligned} & \text { PROCEEDINGS } \\ & \text { OF SCIENCE }\end{aligned}$

\title{
Determination of $|\mathrm{Vub}|$ and $|\mathrm{Vcb}|$ : A Theory Perspective
}

Thomas Mannel*

Siegen University

E-mail: mannel@physik.uni-siegen.de

I discuss some the recent theory developments relevant for the extraction of $V_{c b}$ and $V_{u b}$

Flavor Physics and CP Violation 2010

May 25-29,2010

Turin, Italy

${ }^{*}$ Speaker. 


\section{Introduction}

The the theoretical machinery relevant for the determination of the CKM matrix elements $V_{c b}$ and $V_{u b}$ from both exclusive and inclusive decays has arrived at a very mature state. In particular, the heavy quark expansion (HQE) became an indispensable tool to tackle this problem.

However, the current situation concerning the extracted values for $V_{c b}$ and $V_{u b}$ indicates that there is still a residual problem either in our theoretical tools or in our understanding of the current data. We observe tensions (which has become the "terminus technicus" for a difference between central values at the level of two $\sigma$ 's) between the values determined from exclusive and inclusive decays for both $V_{c b}$ and $V_{u b}$.

In the following I will discuss the theoretical issues relatad to the discussion of these tensions. While the tension in $V_{c b}$ currently tends to decrease, the origin of the tension in $V_{u b}$ remains unclear.

\section{Inclusive $b \rightarrow c$}

One of the most prominent applications of the Heavy Quark Expansion (HQE) is the calculation of the inclusive semileptonic rate. Within HQE, the total semileptonic rate as well as the differential distributions are represented in terms of an expansion of the form [1,32]

$$
\begin{aligned}
d \Gamma= & d \Gamma_{0}+\left(\frac{\Lambda_{\mathrm{QCD}}}{m_{b}}\right)^{2} d \Gamma_{2}+\left(\frac{\Lambda_{\mathrm{QCD}}}{m_{b}}\right)^{3} d \Gamma_{3}+\left(\frac{\Lambda_{\mathrm{QCD}}}{m_{b}}\right)^{4} d \Gamma_{4} \\
& +d \Gamma_{5}\left(a_{0}\left(\frac{\Lambda_{\mathrm{QCD}}}{m_{b}}\right)^{5}+a_{2}\left(\frac{\Lambda_{\mathrm{QCD}}}{m_{b}}\right)^{3}\left(\frac{\Lambda_{\mathrm{QCD}}}{m_{c}}\right)^{2}\right)+\ldots+d \Gamma_{7}\left(\frac{\Lambda_{\mathrm{QCD}}}{m_{b}}\right)^{3}\left(\frac{\Lambda_{\mathrm{QCD}}}{m_{c}}\right)^{4}
\end{aligned}
$$

The coefficients $d \Gamma_{i}$ are themselves functions of $m_{c} / m_{b}$ which are - up to logarithms of $m_{c}$ - regular in the limit $m_{c} \rightarrow 0$, and which have an expansion in $\alpha_{s}\left(m_{b}\right)$. Furthermore, the $d \Gamma_{i}$ depend on nonperturbative parameters corresponding to matrix elements of increasing dimension.

The relevant hadronic matrix elements are

$$
\begin{aligned}
& 2 M_{H} \mu_{\pi}^{2}=-\left\langle H(v)\left|\bar{Q}_{v}(i D)^{2} Q_{v}\right| H(v)\right\rangle: \quad \text { Kinetic Energy } \\
& 2 M_{H} \mu_{G}^{2}=\left\langle H(v)\left|\bar{Q}_{v} \sigma_{\mu v}\left(i D^{\mu}\right)\left(i D^{v}\right) Q_{v}\right| H(v)\right\rangle: \quad \text { Chromomagnetic Moment }
\end{aligned}
$$

for $d \Gamma_{2}$ and

$$
\begin{array}{rlr}
2 M_{H} \rho_{D}^{3} & =-\left\langle H(v)\left|\bar{Q}_{v}\left(i D_{\mu}\right)(i v D)\left(i D^{\mu}\right) Q_{v}\right| H(v)\right\rangle: & \text { Darwin Term } \\
2 M_{H} \rho_{L S}^{3} & =\left\langle H(v)\left|\bar{Q}_{v} \sigma_{\mu v}\left(i D^{\mu}\right)(i v D)\left(i D^{v}\right) Q_{v}\right| H(v)\right\rangle: & \text { Spin-Orbit Term }
\end{array}
$$

for $d \Gamma_{3}$. These four matrix elements can be extraced from the data; the results of the fits have been shown in the talk by R. Kowalewski [3].

The current status of the calculation includes the tree level terms up to and including $d \Gamma_{5}$, while for $d \Gamma_{0}$ the complete $\mathscr{O}\left(\alpha_{s}^{2}\right)$ corrections are known. Furthermore, for $d \Gamma_{2}$ the $\mathscr{O}\left(\alpha_{s}\right)$ corrections are known for the $\mu_{\pi}^{2}$ contribution. The tree level contributions of $d \Gamma_{4}$ and $d \Gamma_{5}$ are not yet included in the experimental fits, but their impact on $V_{c b}$ is very small.

The HQE parameters as well as the quark masses are determined from the data, using moments of the charged lepton energy, the hadronic energy and the hadronic invariant mass. The 


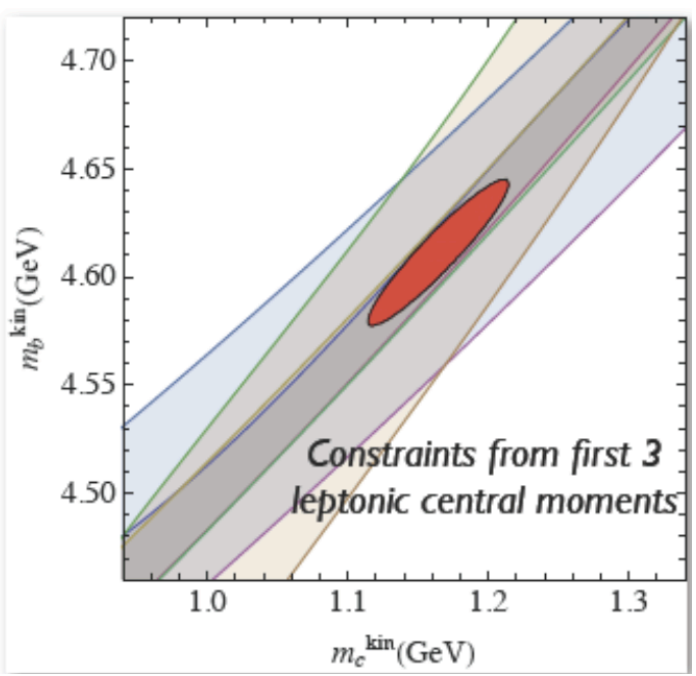

Figure 1: Correlation in the $m_{b}-m_{c}$ plane for the semileptonic moments (Plot taken from P. Gambino).

corresponding fit results are presented in the talk by R. Kowalewski at this conference [3]. One delicate issue in the precision determination of $V_{c b}$ is the issue of quark masses. Althought neither the bottom nor the charm mass are individually known with sufficiently high precision, the rate as well the moments map out a strip in the $m_{b}-m_{c}$ plane, corresponding roughly to the combination $m_{b}-0.7 m_{c}$. As can be inferred from fig. 1, this combination can be extracted from the data of the semileptonic decays with a sufficient accuracy to warrant a final theoretical uncertainty in $V_{c b}$ of about $1.5 \%$.

The size of the radiative corrections is correlated with the choice of the mass definitions, and there two schemes have been proposed which yield a comparable final precision for the theoretical predictions. The so-called kinetic scheme uses a mass definition derived from a sum rule for the kinetic energy [4] while the second scheme is the so-called 1S-scheme where the mass definition is derived from the 1S state of the Upsilon system [5]. The corresponding fits and results have been shown in the talk by R. Kowalewski at this conference [3].

The complete calculation of the QCD radiative corrections have been calculated at order $\alpha_{s}^{2}$ has been performed in [6,7]. The order $\alpha_{s}^{2} \beta_{0}$ contributions are known for some time [1], and it turns out the non-BLM contributions are in fact small. Hence the main impact of the full $\alpha_{s}^{2}$ calculation is a slight reduction of the higher-order uncertainties.

Nonperturbative contributions beyond $1 / m_{b}^{3}$ have been studied up to $1 / m_{b}^{5}$ indicating that their impact is as well small. This requires an estimate of the hadronic matrix elements, which is done on the basis of a "ground state saturation ansatz" decribed in [8]. The contributions to the total rate turn out to be

$$
\left.\delta \Gamma\right|_{1 / m_{b}^{4}} \approx+0.29 \%,\left.\quad \delta \Gamma\right|_{1 / m_{b}^{3}} \approx-2.84 \%,\left.\quad \delta \Gamma\right|_{1 / m_{b}^{2}} \approx-4.29 \%
$$

with $\left.\delta \Gamma\right|_{1 / m_{b}^{i}}=\left(\left.\Gamma\right|_{1 / m_{i}}-\left.\Gamma\right|_{1 / m_{i-1}}\right) / \Gamma_{\text {parton }}$. Thus there is some confidence that the $1 / m_{b}$ expansion, such that a relative theoretical uncertainty of 1-1.5\% in the inclusive determination of $V_{c b}$ has been achieved. 


\section{Exclusive $b \rightarrow c$}

The determination of $V_{c b}$ from exclusive $b \rightarrow c$ transitons is based on the expressions

$$
\begin{aligned}
\frac{d \Gamma}{d \omega}\left(B \rightarrow D^{*} \ell \bar{v}_{\ell}\right) & =\frac{G_{F}^{2}}{48 \pi^{3}}\left|V_{c b}\right|^{2} m_{D^{*}}^{3}\left(\omega^{2}-1\right)^{1 / 2} P(\omega)(\mathscr{F}(\omega))^{2} \\
\frac{d \Gamma}{d \omega}\left(B \rightarrow D \ell \bar{v}_{\ell}\right) & =\frac{G_{F}^{2}}{48 \pi^{3}}\left|V_{c b}\right|^{2}\left(m_{B}+m_{D}\right)^{2} m_{D}^{3}\left(\omega^{2}-1\right)^{3 / 2}(\mathscr{G}(\omega))^{2}
\end{aligned}
$$

where $\omega=v v^{\prime}$ is related to the energy of the outgoing $D^{(*)}$ meson in the $B$-rest frame and $P(\omega)$ is a calculable Phase space factor. The quantities $\left|V_{c b}\right| \mathscr{F}(1)$ and $\left|V_{c b}\right| \mathscr{G}(1)$ are extracted from experiment by extrapolation to the non-recoil point $\omega=1$.

The extraction of $V_{c b}$ hence needs a theoretical prediction for the form factors $\mathscr{F}(\omega)$ and $\mathscr{G}(\omega)$, at least at the non-recoil point. Heavy Quark Symmetries predict theses form factors to be unity at $\omega=1$ in the infinite mass limit for both the bottom and the charm quark. Hence the main point is to calculate the deviations from unity.

There has been substantial progress with lattice calculations over the last few years. Without referring to the infinite mass limit the following unquenched lattice results are quoted $[9,10,11]$

$$
\mathscr{F}(1)=0.927 \pm 0.024 \quad \mathscr{G}(1)=1.074 \pm 0.018 \pm 0.016
$$

After the conference an update has been presented at the CKM 2010 workshop [12]

$$
\mathscr{F}(1)=0.908 \pm 0.016
$$

Alternatively, one may make use of the heavy mass expansion to determine the form factors. Based on the so called BPS limit, in which $\mu_{\pi}^{2}=\mu_{G}^{2}$, one may estimate the form factor $\mathscr{G}(1)$ [13]

$$
\mathscr{G}(1)=1.04 \pm 0.02
$$

The deviation form unity of the form factor $\mathscr{F}(1)$ can be estimated using zero recoil sum rules; the most recent estimate is given in [14]

$$
\mathscr{F}(1)=0.86 \pm 0.04
$$

It is worthwhile to note that the non-lattice estimates are lower by on to two sigmas compared to the lattice estimates; consequently the lattice estimates yield a relatively small value of $V_{c b}$

$$
V_{c b, e x c l}=(38.7 \pm 1.1) \times 10^{-3}, \quad \text { updated to } \quad V_{c b, e x c l}=(39.7 \pm 1.0) \times 10^{-3} \quad \text { at CKM } 2010
$$

while the non-lattice estimates yield

$$
V_{c b, e x c l}=(41.0 \pm 1.5) \times 10^{-3},
$$

which turns out to be more compatible with the inclusive extraction. 


\section{Exclusive $b \rightarrow u$}

The most precise determination of $V_{u b}$ from exclusive decays is performed using the channel $B \rightarrow \pi \ell \bar{v}$, since the relevant form factors can be computed quite reliably, either on the lattice or by QCD sum rules. Determinations based e.g. on $B \rightarrow \rho \ell \bar{v}$ are more difficult, since the relevant form factors cannot be calculated as reliably due to the sizable width of the $\rho$.

To his end, we write the form factor of the $B \rightarrow \pi$ transition as

$$
\left\langle\pi(p)\left|\bar{u} \gamma_{\mu} b\right| B(p+q)\right\rangle=f_{B \pi}^{+}\left(q^{2}\right)(2 p+q)_{\mu}+\text { Terms with } f_{B \pi}^{0}\left(q^{2}\right)
$$

Neglecting the final state lepton masses we get for the rate

$$
\frac{d \Gamma\left(\bar{B}^{0} \rightarrow \pi^{+} \ell^{-} v\right)}{d q^{2}}=\frac{G_{F}^{2}\left|V_{u b}\right|^{2}}{24 \pi^{3}} p_{\pi}^{3}\left|f_{B \pi}^{+}\left(q^{2}\right)\right|^{2}+O\left(m_{\ell}^{2}\right)
$$

where $p_{\pi}$ is the absolute value of the pion three momentum.

The shape of the form factor is well constrained by analyticity, and the absolute normalization can be obtained by lattice and QCD sum rule calculations. In fact. the QCD sum rules work best at $q^{2}=0$, while the lattice calculations prefer values close to the maximum momentum transfer $q^{2}=\left(m_{B}-m_{\pi}\right)^{2}$.

Although there are various parametrizations for the form factor available, the variation of the result for $V_{u b}$ is very small. In fact, using either the Becirevic Kaidalov parametrization [15]

$$
f_{+}\left(q^{2}\right)=\frac{f_{+}(0)}{\left(1-q^{2} / m_{B^{*}}^{2}\right)\left(1-\alpha q^{2} / m_{B^{*}}^{2}\right)}
$$

or a parametrization based on a conformal transformation [16, 17]

$$
P(t) \phi\left(t, t_{0}\right) f_{+}(t)=\sum_{k=0}^{\infty} a_{k}\left(t_{0}\right) z^{k}\left(t, t_{0}\right)
$$

with

$$
z\left(t, t_{0}\right)=\frac{\sqrt{t_{+}-t}-\sqrt{t_{+}-t_{0}}}{\sqrt{t_{+}-t}+\sqrt{t_{+}-t_{0}}}, \quad t_{+}=\left(m_{B}+m_{\pi}\right)^{2}
$$

yield form factors which are pratically identical. In combination with the precise measurements an accurate determination of $V_{u b}$ can be performed.

Combining the results from light-cone sum rules, the measurements of the shape of the form factor and from lattice QCD and using the (slightly modified) series parametrization (4.4) [18], one finds the form factor shape as shown in fig. 2. Table 1 gives a sample of the recent results on $V_{u b}$ using different models and different inputs.

The current theoretical uncertainty on the form factor is estimated to be slightly less than $10 \%$, and hence the variation in the central values is covered by the theory uncertainties.

\section{Inclusive $b \rightarrow u$}

Unlike for $V_{c b}$, inclusive determinations of $V_{u b}$ can in general not rely on the local version of the OPE, since the phase space cuts needed to suppress the background from charmed states render 


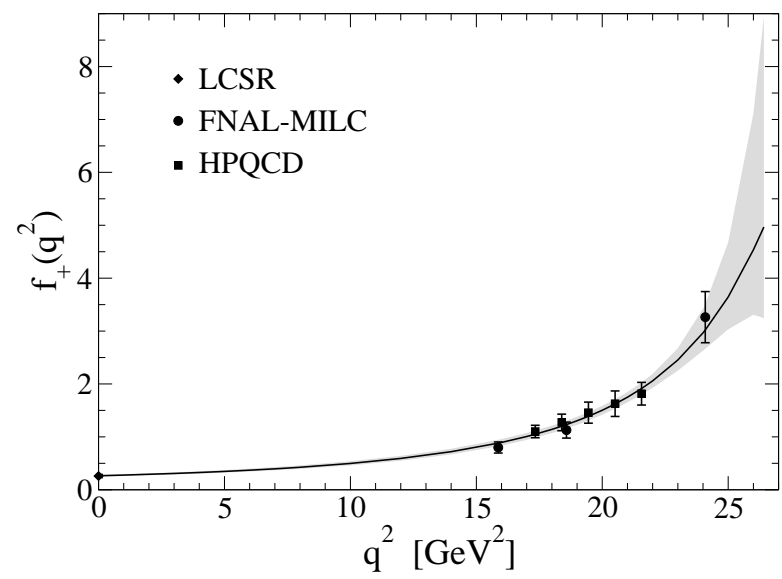

Figure 2: Combined result for the form factor $f_{+}$(figure taken from [18]).

\begin{tabular}{|c|c|c|c|}
\hline [Ref.] & $\begin{array}{c}f_{B \pi}^{+}\left(q^{2}\right) \\
\text { calculation }\end{array}$ & $\begin{array}{c}f_{B \pi}^{+}\left(q^{2}\right) \\
\text { input }\end{array}$ & $\left|V_{u b}\right| \times 10^{3}$ \\
\hline FNAL-MILC [19] & lattice & - & $3.38 \pm 0.35$ \\
\hline HPQCD [20] & lattice & - & $3.55 \pm 0.25 \pm 0.50$ \\
\hline Ball, Zwicky [21] & LCSR & - & $3.5 \pm 0.4 \pm 0.1$ \\
\hline Flynn, Nieves [22] & - & lattice $\oplus$ LCSR & $3.47 \pm 0.29 \pm 0.03$ \\
\hline Duplancic et al. [23] & LCSR & - & $3.5 \pm 0.4 \pm 0.2 \pm 0.1$ \\
\hline $\begin{array}{c}\text { Bourrely, Caprini, } \\
\text { Lellouch [18] }\end{array}$ & - & lattice $\oplus$ LCSR & $3.54 \pm 0.24$ \\
\hline
\end{tabular}

Table 1: A sample of recent results for $V_{u b}$ from $B \rightarrow \pi \ell \bar{v}$.

this version of the OPE invalid. In turn, methods relying on the local OPE suffer from only small data samples.

The version of the OPE that can be used in the case at hand is analogous to deep inelastic scattering, i.e an expansion in twists. Like in deep inelastic scattering light-cone distribution functions appear, which parametrize the necessary non-perturbative input. Thus, instead of the HQE parameters we have to deal with non-perturbative functions. The systematic way to formulate this expansion is "soft collinear effective theory", which provides the framework for the calculation of radiative corrections.

Several ways have been proposed to obtain the distribution functions. Since they are universal, they may also be obtained from other heavy-to-light decays, the most prominent one is $B \rightarrow X_{s} \gamma$. However, at subleading level several distribution functions appear, which cannot be extracted by a simple comparison with $B \rightarrow X_{s} \gamma$.

The moments of the distribution functions are matrix elements of local operators which may be related to the heavy quark expansion parameters appearing in the OPE for $V_{c b}$. Hence another option is to model the distribution functions under the constraint that the moments coincide with the ones obtained form the data of $B \rightarrow X_{c} \ell \bar{v}$ such as the BLNP [24] and GGOU [25] approaches. The most sophisticated approach along this line is the SIMBA project [26], where the distribution 
functions are expanded in terms of basis functions. Finally, one may also adopt a more model dependent approach $[27,28]$; however, here it is much more difficult to get a reliable estimate on the uncertainties.

The results of the corresponding fits have been shown in the talk by R. Kowalewsky at this conference [3]. The values of $V_{u b}$ from the inclusive determinations turn out to be systematically larger than the values obtained from $B \rightarrow \pi \ell \bar{v}$. However, depending on the way to assign and add uncertainties this may still be considered insignificant. Nevertheless, this has triggered speculations on possible new physics effects in the $b \rightarrow u$ charged current (see e.g. [29]), but also a more deep scrutiny of the standard model calculation.

This tension has motivated work on NNLO corrections to the current determinations. In the framework of BLNP a NNLO calculation has been performed [30], which indicates a substantial size of the NNLO corrections, at least in the BLNP scheme. In fact, for the various cuts on lepton energy as well as on the hadronic invariant mass one obtains shifts of the extracted $V_{u b}$ value of the order of $10-15 \%$. However, the shift is positive and hence make the tension between the inclusive and exclusive even worse.

The second source that has been scrutinized as a possible source for this tension is weak annihilation $[31,32]$. It is well known also from lifetime calculations that at order $1 / m_{b}^{3}$ contributions of four-quark operators of the form

$$
\frac{d \Gamma}{d m_{X}^{2}} \sim\left\langle B\left|\bar{b} \Gamma q \bar{q} \Gamma^{\dagger} b\right| B\right\rangle \delta\left(m_{X}^{2}\right)
$$

appear, which are concentrated at low hadronic masses. For $q=c$ these have been named "intrinsic charm", while for $q=u$ these are called "weak annihilation contributions". These four quark matrix elements mix under renormalization with the Darwin term, compensating its $\mu$ dependence.

There are two contributions to the four quark matrix elements. The "valence quark independent" (VQI) piece is the same for both $B^{+}$and $B^{0}$, while the "valence quark dependent" (VQD) part creates a difference between $B^{+}$and $B^{0}$. Hence the VQI contribution may be identified with "intrinsic charm" in the formal limit $m_{c} \rightarrow 0$, while the VQD piece can be inferred from corresponding $D$ decays.

Starting from the results for intrinsic charm one may invent a simple model parametrization for the VQI part of weak annihilation. As a simple model we may use

$$
\left\langle B\left|\bar{b}_{L} \gamma_{\mu} q_{L} \bar{q}_{L} \gamma_{v} b_{L}\right| B\right\rangle \sim \frac{\rho_{D}^{3}}{4 \pi^{2}} \ln \left(\frac{\mu^{2}}{M_{*}^{2}+m_{q}^{2}}\right)=\left\{\begin{array}{l}
\frac{\rho_{D}^{3}}{4 \pi^{2}}\left(\ln \left(\frac{\mu^{2}}{m_{q}^{2}}\right)-\frac{M_{*}^{2}}{m_{q}^{2}}+\mathscr{O}\left(1 / m_{q}^{4}\right)\right) \\
\frac{\rho_{D}^{3}}{4 \pi^{2}} \ln \left(\frac{\mu^{2}}{M_{*}^{2}}\right)+\mathscr{O}\left(m_{q}^{2}\right)
\end{array}\right.
$$

where the parameter $M^{*}$ can be obtained from the higher-order matrix elements appearing in the HQE [8]. Extrapolating $m_{c} \rightarrow 0$ we obtain at $\mu=0.7 \mathrm{GeV}$ the estimate

$$
\frac{\delta \Gamma^{V Q I}}{\Gamma_{s l}} \approx-0.015
$$

The VQD contribution can be estimated from the data on $D$ decays; scaling up to the bottom mass yields (see also [33])

$$
\frac{\Gamma\left(B^{+} \rightarrow X \ell \bar{v}_{\ell}\right)-\Gamma\left(B^{0} \rightarrow X \ell \bar{v}_{\ell}\right)}{\Gamma\left(B \rightarrow X \ell \bar{v}_{\ell}\right)}=-(0.005 \ldots .0 .01)
$$


From these estimates there is no hint that weak annihilation could explain the tension between exclusive and inclusive determinations of $V_{u b}$. It would be interesting to verify these estimates by a measurement of the rates for $B^{+}$and $B^{0}$ in the region of small hadronic masses.

\section{Conclusions}

Due to heavy quark expansion and heavy quark effective theory, but also due to lattice calculations and QCD sum rules the theoretical machinery for the determinations of $V_{c b}$ and $V_{u b}$ is in a mature state, resulting in an expected relative theoretical uncertainty ranging from approximatlely $1.5 \%$ for inclusive $V_{c b}$ to approximately $10 \%$ for exclusive $V_{u b}$.

Despite of this, certain tensions appear in the results for these CKM matrix elements. The determination of exclusive $V_{c b}$ relies on lattice calculations of the form factors at the non-recoil point, which systematically lead to smaller central values of $V_{c b}$ compared to the inclusive determinations, yet with an agreement within uncertainties. However, estimates based on QCD sum rules at zero recoil indicate smaller values for the form factors, resulting in larger values for $V_{c b}$ with better compatibility. In the meantime, smaller form factors have also been given by lattice groups at CKM 2010; my personal conclusion is that the tension between inclusive and exclusive determinations of $V_{c b}$ tends to disappear.

More severe is currently the tension in the case of $V_{u b}$. The exclusive determination from the semi-leptonic $B \rightarrow \pi$ decay is quite mature, since the various calculations of the form factor yield a consistent picture, pinning down the form factor quite precisely. In combination with a significant amount of data a reliable determination of $V_{u b}$ becomes possible. On the other side, the theory input for inclusive decays also seems in a mature state, although higher twist contributions are hard to estimate. Still the precision in this determination is at least comparable to the exclusive determination based on $B \rightarrow \pi \ell \bar{v}$. Nevertheless, the exclusive determinations systematically lead to smaller central values compared to the inclusive ones; however, again within uncertainties. Unlike for $V_{c b}$ the current tendency is that new data analyses and new calculations make the situation even worse; the NNLO calculations for the inclusive method seems to drive the central value of $V_{u b}$ further up, and determinations based on vector-meson final states $B \rightarrow \rho \ell \bar{v}$ and $B \rightarrow \omega \ell \bar{v}$ yield even smaller central values. This has triggered speculations on possible new physics effects, but in total the situation concerning $V_{u b}$ is currently unclear.

\section{References}

[1] D. Benson, I. I. Bigi, T. Mannel and N. Uraltsev, Nucl. Phys. B 665 (2003) 367 [arXiv:hep-ph/0302262].

[2] I. Bigi, T. Mannel, S. Turczyk and N. Uraltsev, JHEP 1004 (2010) 073 [arXiv:0911.3322 [hep-ph]].

[3] R. Kowalewski, Measurement of $\left|V_{c b}\right|$ and $\left|V_{u b}\right|$, talk presented at this conference.

[4] I. I. Y. Bigi, M. A. Shifman, N. G. Uraltsev and A. I. Vainshtein, Phys. Rev. D 52 (1995) 196 [arXiv:hep-ph/9405410].

[5] A. H. Hoang, Z. Ligeti and A. V. Manohar, Phys. Rev. D 59 (1999) 074017 [arXiv:hep-ph/9811239].

[6] K. Melnikov, Phys. Lett. B 666 (2008) 336 [arXiv:0803.0951 [hep-ph]]. 
[7] M. Dowling, A. Pak and A. Czarnecki, Phys. Rev. D 78 (2008) 074029 [arXiv:0809.0491 [hep-ph]].

[8] T. Mannel, S. Turczyk and N. Uraltsev, JHEP 1011 (2010) 109 [arXiv:1009.4622 [hep-ph]].

[9] C. Bernard et al., Phys. Rev. D 79, 014506 (2009) [arXiv:0808.2519 [hep-lat]].

[10] G. T. . Bodwin, 22nd International Symp. on Lattice Field Theory, Batavia, (Ill.), 21-26 Jun 2004.

[11] G. M. de Divitiis, R. Petronzio and N. Tantalo, Nucl. Phys. B 807 (2009) 373 [arXiv:0807.2944 [hep-lat]].

[12] P. Mackenzie, talk presented at CKM2010, Warwick, Sep. 6-10, 2010.

[13] N. Uraltsev, Phys. Lett. B 585 (2004) 253 [arXiv:hep-ph/0312001].

[14] P. Gambino, T. Mannel and N. Uraltsev, Phys. Rev. D 81 (2010) 113002 [arXiv:1004.2859 [hep-ph]].

[15] D. Becirevic and A. B. Kaidalov, Phys. Lett. B 478 (2000) 417 [arXiv:hep-ph/9904490].

[16] M. C. Arnesen, B. Grinstein, I. Z. Rothstein and I. W. Stewart, Phys. Rev. Lett. 95, 071802 (2005) [arXiv:hep-ph/0504209].

[17] C. G. Boyd, B. Grinstein and R. F. Lebed, Phys. Rev. D 56 (1997) 6895 [arXiv:hep-ph/9705252].

[18] C. Bourrely, I. Caprini and L. Lellouch, Phys. Rev. D 79 (2009) 013008 [Erratum-ibid. D 82 (2010) 099902] [arXiv:0807.2722 [hep-ph]].

[19] J. A. Bailey et al., lattice Phys. Rev. D 79 (2009) 054507 [arXiv:0811.3640 [hep-lat]]; C. Bernard et al., Phys. Rev. D 80 (2009) 034026 [arXiv:0906.2498 [hep-lat]].

[20] E. Dalgic, A. Gray, M. Wingate, C. T. H. Davies, G. P. Lepage and J. Shigemitsu, Phys. Rev. D 73 (2006) 074502 [Erratum-ibid. D 75 (2007) 119906] [arXiv:hep-lat/0601021].

[21] P. Ball and R. Zwicky, Phys. Rev. D 71 (2005) 014015 [arXiv:hep-ph/0406232].

[22] J. M. Flynn and J. Nieves, Phys. Rev. D 76 (2007) 031302 [arXiv:0705.3553 [hep-ph]].

[23] G. Duplancic, A. Khodjamirian, T. Mannel, B. Melic and N. Offen, JHEP 0804 (2008) 0140 [arXiv:0801.1796 [hep-ph]].

[24] B. O. Lange, M. Neubert and G. Paz, Phys. Rev. D 72 (2005) 073006 [arXiv:hep-ph/0504071].

[25] P. Gambino, P. Giordano, G. Ossola and N. Uraltsev, JHEP 0710 (2007) 058 [arXiv:0707.2493 [hep-ph]].

[26] F. U. Bernlochner et al., arXiv:1011.5838 [hep-ph].

[27] J. R. Andersen and E. Gardi, JHEP 0601 (2006) 097 [arXiv:hep-ph/0509360].

[28] U. Aglietti, F. Di Lodovico, G. Ferrera and G. Ricciardi, Eur. Phys. J. C 59 (2009) 831 [arXiv:0711.0860 [hep-ph]].

[29] A. Crivellin, Phys. Rev. D 81 (2010) 031301 [arXiv:0907.2461 [hep-ph]].

[30] C. Greub, M. Neubert and B. D. Pecjak, Eur. Phys. J. C 65 (2010) 501 [arXiv:0909.1609 [hep-ph]].

[31] C. Breidenbach, T. Feldmann, T. Mannel and S. Turczyk, Phys. Rev. D 78 (2008) 014022 [arXiv:0805.0971 [hep-ph]].

[32] I. Bigi, T. Mannel, S. Turczyk and N. Uraltsev, JHEP 1004 (2010) 073 [arXiv:0911.3322 [hep-ph]].

[33] L. Kamenik, talk at this conference 